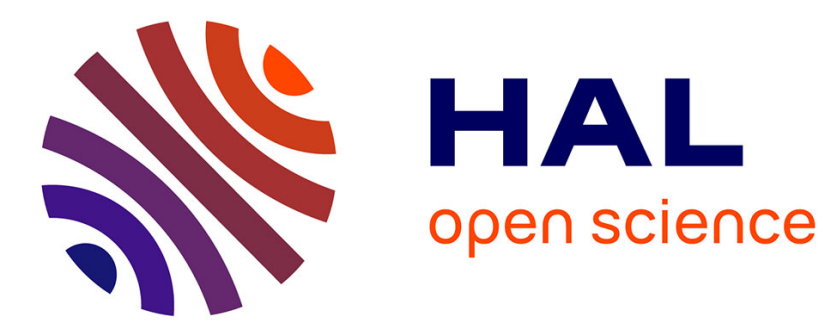

\title{
Synthesis Method for Manifold-Coupled Multiplexers
}

David Martínez Martínez, Stéphane Bila, Fabien Seyfert, Martine Olivi, Olivier Tantot, Ludovic Carpentier

\section{To cite this version:}

David Martínez Martínez, Stéphane Bila, Fabien Seyfert, Martine Olivi, Olivier Tantot, et al.. Synthesis Method for Manifold-Coupled Multiplexers. EuMC 2019 - 49th European Microwave Conference, Oct 2019, Paris, France. hal-02377002

\section{HAL Id: hal-02377002 \\ https://hal-unilim.archives-ouvertes.fr/hal-02377002}

Submitted on 20 Jan 2020

HAL is a multi-disciplinary open access archive for the deposit and dissemination of scientific research documents, whether they are published or not. The documents may come from teaching and research institutions in France or abroad, or from public or private research centers.
L'archive ouverte pluridisciplinaire HAL, est destinée au dépôt et à la diffusion de documents scientifiques de niveau recherche, publiés ou non, émanant des établissements d'enseignement et de recherche français ou étrangers, des laboratoires publics ou privés. 


\title{
Synthesis Method for Manifold-Coupled Multiplexers
}

\author{
D. Martínez Martínez ${ }^{\# \star 1}$, S. Bila ${ }^{\# 2}$, F. Seyfert ${ }^{\star 3}$, M. Olivi ${ }^{\star 4}$, O. Tantot $^{\# 5}$, L. Carpentier ${ }^{\dagger 6}$ \\ \#Université de Limoges, CNRS, XLIM, UMR7252, F-87000 Limoges \\ *Inria Sophia Antipolis Méditerranée, 2004 Route des Lucioles, 06902 Valbonne \\ ${ }^{\dagger}$ CNES Centre National d'études spatiales, 18 Avenue Edouard Belin, 31400 Toulouse \\ $\left\{{ }^{1}\right.$ david.martinez, ${ }^{3}$ fabien.seyfert, ${ }^{4}$ martine.olivi $\} @$ inria.fr, $\left\{{ }^{2}\right.$ stephane.bila, ${ }^{5}$ olivier.tantot $\} @$ xlim.fr \\ ${ }^{6}$ ludovic.carpentier@cnes.fr
}

\begin{abstract}
This paper presents a novel synthesis approach for manifold-coupled multiplexers. This technique provides, on one side, the manifold structures to avoid dealing with manifold peaks, and on the other side, the circuit model of the channel filters to be connected to the manifold. Furthermore, to show the validity of the proposed approach, a plastic-printed prototype of a triplexer is presented.
\end{abstract}

Keywords - multiplexers, matching filters synthesis, manifold.

\section{INTRODUCTION}

In general, multiplexer synthesis involves dealing with manifolds peaks (spikes) appearing inside the passbands. Those are narrow transmission zeros that appear due to phase recombinations happening inside the manifold. In the design of multiplexers, we often face the problem of avoiding these peaks and there are many contributions to this problem in the literature [1], [2], most of them providing heuristic procedures to solve or simplify the problem. Nevertheless, these procedures usually apply after the design is completed and an EM simulation reveals the presence of the peaks.

In this paper, we propose a rigorous approach to the synthesis of multiplexers based on functional analysis. First, the transmission lines composing the manifold are determined to avoid manifold peaks appearing within the passbands by using an estimation of the out-of-band phase of each filter. Secondly, the channel filters are calculated by a continuation algorithm which in this case replaces the circuit optimization. This procedure also decouples the manifold design from the filter synthesis. In addition, no overall optimization of the multiplexer is required, only an isolated optimization of each filter is performed.

The presented approach apply to a generic multiplexer, however to illustrate the procedure we use the example of a triplexer with a channel of $4.4 \%$ bandwidth and two channels with $2 \%$ relative bandwidth ${ }^{1}$.

\section{MANIFOLD SYNTHESIS}

We address now the problem of manifold peaks, why they appears and how to avoid them. Let $J$ be the scattering matrix of the junction (manifold) with a zero-based port numbering.

\footnotetext{
${ }^{1}$ Due to confidentiality reasons, there are no indications relative to the size or working frequency of the presented device.
}

Additionally we denote by $f_{i}$ the output reflection (parameter $S_{22}$ ) of the $i$-th channel filter and by $M$ the scattering matrix of the whole multiplexer. This notation is illustated in Fig. 1 where the key scattering parameters are indicated.

Next we use a divide and conquer strategy. Note that manifold peaks are transmission zeros from port $i \in\{1,2,3\}$ to port 0 of the manifold within the band of the $i$-th filter respectively. We use a model based of T-junctions (3-port elements) connected by transmission lines (Fig. 1). With this model, transmission zeros from channel ports to the commun port can not be introduced by the transmission lines since they only modify the phase of the signal. Therefore transmission zeros happen inside the $T$ elements. For instance, if there is a transmission zero from channel 3 to the commun port at a given frequency, then either $T_{2}$ or $T_{1}$ (after channel filters 2 and 3 are connected), or both introduce a transmission zero at that frequency. Conversely, if $T_{1}$ or $T_{2}$ presents a transmission zero in the band of the filter 3 , then a manifold peak will certainly appear at that frequency.

\section{A. Transmission Zeros in 3-Port Devices}

To determine how the manifold peaks are produced, it is important to understand when a 3-port device presents a transmission zero between two of its terminals. Let $S(\omega)$ be the scattering matrix of a generic 3-port device. If port 3 of $S$ is closed by a load with reflection coefficient $F(\omega)$ (denoted by the operation $S \circ F$ ) the following $2 \times 2$ matrix is obtained

$$
S \circ F=\left[\begin{array}{ll}
S_{11} & S_{12} \\
S_{21} & S_{22}
\end{array}\right]+\left[\begin{array}{ll}
S_{13} S_{31} & S_{13} S_{32} \\
S_{23} S_{31} & S_{23} S_{32}
\end{array}\right] \frac{F}{1-F S_{33}}
$$

First we consider that the $T$-element itself does not have transmission zeros at finite frequencies. Then we compute the value of $F$ such that the previous matrix has a transmission zero at a given frequency. Assuming the matrix $S$ is reciprocal, we impose a transmission zero between terminals 2 and 1 by equating the 12 element to zero

$$
\begin{aligned}
& S_{12}+\frac{S_{13} S_{32} F}{1-F S_{33}}=0 \\
& \frac{-S_{12}}{S_{13} S_{32}-S_{12} S_{33}}=F
\end{aligned}
$$

Note that the previous denominator is the 21 element of the cofactor matrix of $S$. Additionally the 12 element of $S^{-1}$ is 
expressed as $\left[S^{-1}\right]_{12}=\frac{C_{21}}{\operatorname{det}(S)}$ where $C$ is the cofactor matrix. Therefore we have

$$
\frac{-S_{12}}{\left[S^{-1}\right]_{12} \operatorname{det}(S)}=F
$$

Finally, we consider the matrix $S$ to be lossless, this implies, together with the reciprocity, $S^{-1}=\bar{S}$ for all $\omega \in \mathbb{R}$. Then

$$
\frac{-S_{12}}{\overline{S_{21}} \operatorname{det}(S)}=F
$$

As a result, when port 3 of the 3-port device is closed by a load with reflection $F$ a transmission zero appears from port 1 to port 2. From a physical point of view, reflection $F$ introduces a virtual short-circuits in the 3-port device.

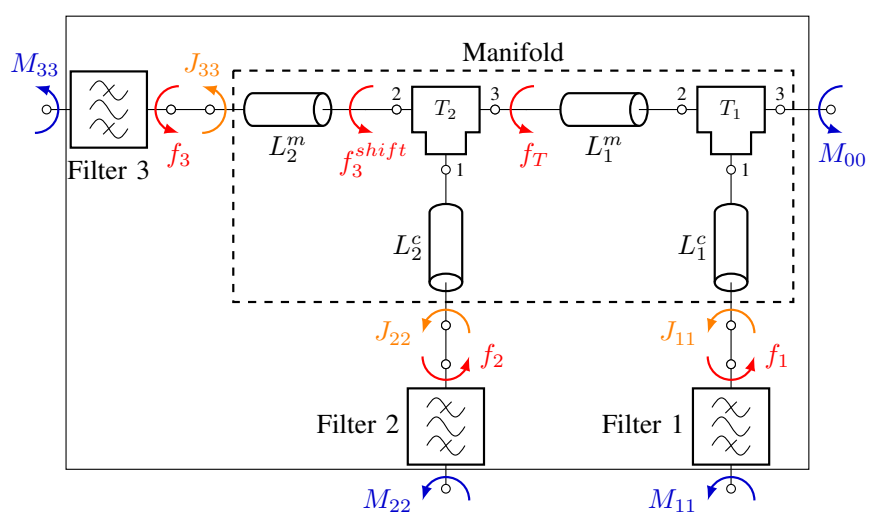

Fig. 1. Triplexer schematic

\section{B. Reference filters}

In order to properly adjust the transmission lines in Fig. 1 it is necessary to know the output reflection of each channel filter namely $f_{1}, f_{2}, f_{3}$. However this information is not yet available. As an alternative, we design a reference filter implementing a Tchebyshev response in each of the channel (Fig. 2). The reference filters are implemented in the same technology as the final filters. In this case a waveguide implementation with a fully inductive topology is used. The later consists of four in-line cavities, two using resonant mode $T E_{101}$ and two others with higher order modes $T E_{102}$ and $T E_{201}$. This structure allows 2 transmission zeros and 6 reflection zeros per filter. Then we make the assumption that the out of band phase of the Tchebyshev filters is not modified much when the in-band reflection is modified from the Tchebyshev response to the final one.

\section{Calculation of Manifold Transmission Lines}

Using the previous theory, we can determine the lengths of the transmission lines in Fig. 1 that minimise the risk of encountering a manifold peak in any of the channel passbands. Note that, in order not to have a transmission zero in the band of channel 3 , elements $T_{1}$ and $T_{2}$ must not introduce any transmission zero in that band. Similarly, $T_{1}$ must not introduce a transmission zero in band 2 . In this example both $T_{1}$ and $T_{2}$ are equal. Thus we just need to compute the reflection that

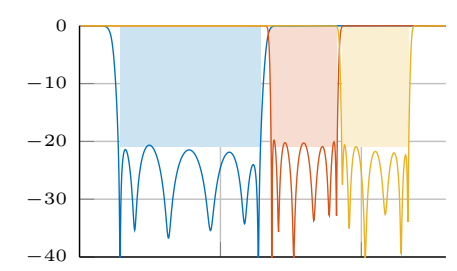

Frequence

(a) Modulus (dB)

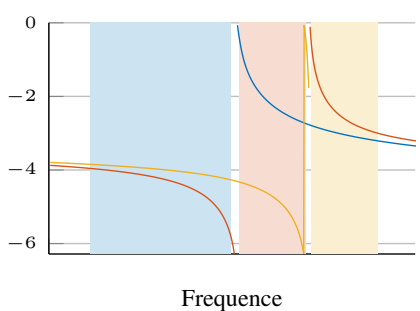

(b) Out-of-band phase (rad)
Fig. 2. Output reflection of the reference filters.

short-circuit $T_{1}$ and $T_{2}$ from terminal 1 (denoted $P_{1}(\omega)$ ) and from the termial $2\left(P_{2}(\omega)\right)$

$$
P_{2}(\omega)=\frac{-S_{13}}{\overline{S_{31}} \operatorname{det}(S)} \quad P_{1}(\omega)=\frac{-S_{32}}{\overline{S_{23}} \operatorname{det}(S)}
$$

The process to determine the transmission lines consists in two different steps:

First the lines $L^{c}$ (vertical transmission lines in Fig. 1). We consider the transmission along the main (horizontal) branch of the manifold. The junction $T_{1}$ must allow the transmission from terminal 3 to 2 in the bands of channels 2 and 3 , meanwhile transmission from terminal 3 to 2 of $T_{2}$ in band 3 must be possible. Transmission lines $L_{1}^{c}$ and $L_{2}^{c}$ are used to shift the phase of the reflections $f_{1}$ and $f_{2}$ such that the phase showed at terminal 1 of $T_{1}$ does not coincide with $P_{1}(\omega)$ withing the bands 2 and 3 . Equivalently the phase brought to terminal 1 of $T_{2}$ must not coincide with $P_{1}(\omega)$ in the third band. We can see in Fig. 3a that the phase of $f_{1}(\omega)$ and $f_{2}(\omega)$ does not coincide with $P_{1}(\omega)$ within the bands of interest. Therefore a minimal length is choosen for $L_{1}^{c}$ and $L_{2}^{c}$.

Second the lines $L^{m}$ (horizontal transmission lines in Fig. 1). After $L_{1}^{c}$ and $L_{2}^{c}$ are selected and reference filters 1 and 2 connected, we can find $L_{2}^{m}$ and $L_{1}^{m}$. We can see in Fig. $3 \mathrm{~b}$ that $f_{3}(\omega)$ coincides with $P_{2}(\omega)$ in the band of channel 2 . Therefore $T_{2}$ will introduce a transmission zero from terminal 3 to 1 and a manifold peak will appear in channel 2. To avoid this problem, reflection $f_{3}$ is shifted by means of the line $L_{2}^{m}$ obtaining the reflection $f_{3}^{\text {shift }}$ (Fig. 1 and 3 b) which does not intersect $P_{2}$ withing the band of interest. Finally we verify that $f_{T}$ (the reflection of the load seen from port 2 of $T_{1}$ ) does not coincide with $P_{2}$ as we can see in Fig. 3b. A minimal length is selected then for $L_{1}^{m}$.

Note that in the case of a N-channel multiplexer, this second step needs to be performed from left to right, starting from the oposite end of the commun port and avancing toward the later one. We need to verify here that any of the $T$ segments blocks the transmission to their correspondent channel.

\section{FILTER SYNTHESIS}

Next, we tackle the synthesis of the channel filters as a matching problem in which the $i$-th filter is matched to the impedance shown by the rest of the multiplexer (including the other filters) in the $i$-th band. In this framework, an analytic 


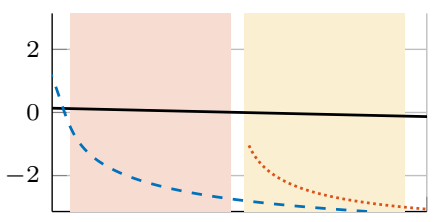

Frequence

(a) $P_{1}:$ solid; $f_{1}:$ dashed; $f_{2}:$ dotted

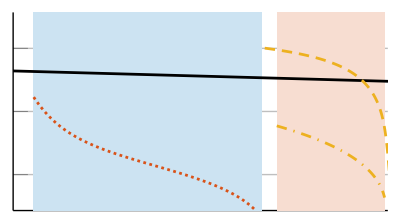

Frequence

(b) $P_{2}$ : solid; $f_{3}:$ dashed; $f_{3}^{\text {shift }}$ : dash-dotted; $f_{T}$ : dotted
Fig. 3. Relevant phases to adjust transmission lines (rad).

synthesis algorithm for matching filters was presented in [3] allowing to have $N$ matching points in a given band. In this paper, we generalize this synthesis technique based on the continuation of an arbitrary solution, for the design of the multiplexers. In this case, the manifold is fixed using the lengths of the transmission lines previously obtained, and the filters are computed to be matched simultaneously. Furthermore the matching points are also fixed in ahead. This algorithm replaces the circuit optimization and makes it possible to obtain the circuit model of the channel filters without modifying the manifold.

\section{A. Belevitch Form of Scattering Matrices}

We introduce now the class of functions we are interested in, namely rational $2 \times 2$ matrices in the Belevitch form

$$
S=\frac{1}{q}\left(\begin{array}{cc}
\epsilon p^{\star} & -\epsilon r^{\star} \\
r & p
\end{array}\right)
$$

with $\epsilon$ a uni-modular constant, and $q, p, r$ polynomials of degree $N$ satisfying $q q^{\star}=p p^{\star}+r r^{\star}$ with $p^{\star}(\omega)=\overline{p(\bar{\omega})}$. Moreover $q$ is a stable (Hurwitz) polynomial.

This parametrisation is customary in classical filter design where the Belevitch form is used to parametrise lossless devices. For instance, we find the same parametrisation applied to multiplexer design in [4]. Similarly the transmission zeros are often set to given positions on the frequency axis with the purpose of increasing the out-of-band selectivity. Note that, if the transmission polynomial $r$ is fixed, then it is possible to parametrise the matrix $S$ only by the numerator polynomial $p$, while expression $q q^{\star}=p p^{\star}+r r^{\star}$ determines the polynomial $q$. In this work we assume polynomial $q$ to be normalised such that its leading term is real and we parametrise $f_{i}=f\left(p_{i}\right)$ with $i \in\{1,2,3\}$.

\section{B. Filter Synthesis as a Simultaneous Matching Problem}

Let us denote by $J$ the scattering matrix of the manifold whose frequency behaviour is already known. For each channel, we fix the polynomial $r_{i}$ having roots at the left and right of the $i$-th passsband. Additionally, a set of matching points $\xi_{i, m}$ is distributed in each passband with $i \in\{1,2,3\}$, $1 \leq m \leq m_{i}$, where $m_{i}$ is the degree of the $i$-th channel filter; in this case $m_{i}=6$ for all $i$.

The objective then is to find the output reflection of each channel filter $f_{i}$ such that $M_{i i}\left(\xi_{i, m}\right)=0$. This implies $f_{i}\left(\xi_{i, m}\right)=\overline{L_{i}\left(\xi_{i, m}\right)}$ where $L_{i}$ the reflection of the load at port 2 as it is explained in [3]. In this case the load includes as well other filters as despicted in Fig. 4 for channel 3. The problem to solve is then stated as:

Problem 3.1 (Simultaneous matching): Find the set of polynomials $P=\left[p_{1}, p_{2}, \ldots, p_{N}\right]$, such that:

$$
f\left(p_{i}\right)\left[\xi_{i, m}\right]-\overline{L_{i}(P)\left[\xi_{i, m}\right]}=0 \quad i \in[1, N] \quad m \in\left[1, m_{i}\right]
$$

where $f\left(p_{i}\right)$ is the output reflection of filter $i$

$$
f\left(p_{i}\right)[\omega]=\frac{p_{i}}{q\left(p_{i}, r_{r}\right)}[\omega]
$$

and $L_{i}(P)$ the load seen by filter $i$ when the other filters are connected to the manifold. Defining the row vector $V_{i}$ as

$$
V_{i}=\left[J_{i, k}\right] \quad k \in[1, N] \quad k \neq i
$$

and $W_{i}$ the submatrix of $J$ where the rows and columns with index 0 and $i$ are removed, we compute $L_{i}(P)$ as

$$
L_{i}(P)=J_{i i}+V_{i}\left[I-F_{i}(P) W_{i}\right]^{-1} F_{i}(P) V_{i}^{T}
$$

with $F_{i}(P)$ the diagonal matrix

$$
F_{i}(P)=\operatorname{diag}\left(\left\{f\left(p_{k}\right)\right\}\right) \quad k \in[1, N] \quad k \neq i
$$

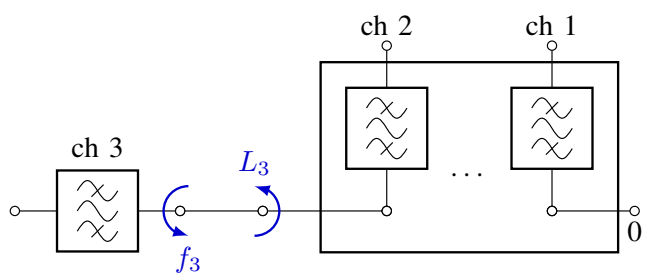

Fig. 4. Synthesis of filter 3 to match the rest of the multiplexer.

\section{A Continuation Algorithm to Obtain the Channel Filters}

To perform the continuation, we add a complex parameter $\lambda_{i, m}$ for each $\xi_{i, m}$ in the expression of $L_{i}(P)\left[\xi_{i, m}\right]$ obtaining

$$
L_{i, m}(P)=J_{i i}+\lambda_{i, m} V_{i}\left[I-F_{i}(P) W_{i}\right]^{-1} F_{i}(P) V_{i}^{T}
$$

evaluated at $\xi_{i, m}$.

Note that for $\lambda_{i, m}=0$ we have $L_{i, m}(P)=J_{i i}\left(\xi_{i, m}\right)$ for which the solution to the problem is trivial. The objective then is to continuate this initial solution, varying these parameters from $\lambda_{i, m}=0$ to $\lambda_{i, m}=1$ for all $i, m$ in small increments $\Delta \lambda_{i, m}$. At each step, the increment to the polynomials $p_{i}$ (denoted by $\Delta p$ ) is computed by

$$
\Delta p=\left[\mathrm{D}_{p_{k}} \lambda_{i, m}\right]^{-1} \Delta \lambda_{i, m}
$$

where $\mathrm{D}_{p_{k}} \lambda_{i, m}$ is the jacobian of the vector of $\lambda_{i, m}$ with respect to parameters $p_{k}$. Note here that when $\lambda_{i, m}=1$, the filters with output reflections $\left[f_{1}, \cdots, f_{N}\right]$ are perfectly matched to the manifold simultaneously at the points $\xi_{i, m}$. Additionally, it should be noted that the path from $\lambda_{i, m}=0$ to $\lambda_{i, m}=1$ is of relevance here since accidents can happen during the continuation (points where the jacobian matrix is singular). In this case, we can modify the trajectory to follow in order to avoid such problematic points. 


\section{RESULTS}

The circuit model of each filter is obtained using the presented method. The computation takes an average time of one second in a laptop with CPU i7-6600U under the environment Matlab R2019a. The obtained circuit is used now for the EM-design of the waveguide filters, which avoids optimizing the whole structure. We show in Fig. 5 the result of each filter optimisation compared to the goal provided by the presented prodedure. These optimisations carried out with the help of the EM-simulation software Microwave Wizard. Finally, we perform a global EM simulation of the triplexer using the software Ansys Electronic Desktop. Figure 7 shows the result obtained with our algorithm and the response resulting from an EM simulation.

A prototype of the presented design has been realised by additive manufacturing. This device, shown in Fig. 6, has a plastic body while the inside has been metalised by silver painting. This technique has already been used in the literature, for instance in [5]. The per-channel reflection and transmission parameters after tuning the structure are shown in Fig. 5. As it can be noticed, the measured response shows a frequency shift toward lower frequencies of about $10 \%$ the total bandwidth. This fact is due to the manufacturing tolerances that could not be compensated by means of the tuning screws when the response is shifted downwards in frequency.
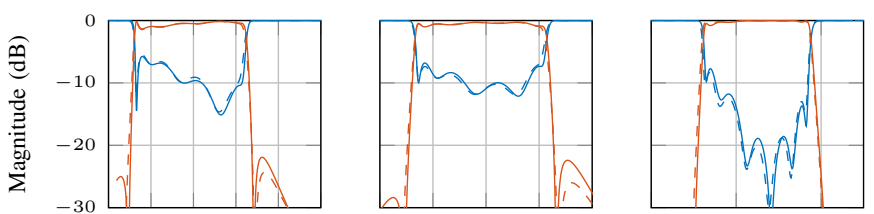

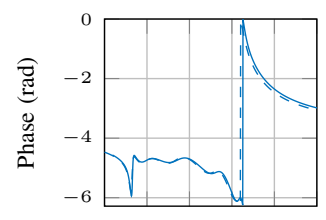

Frequence

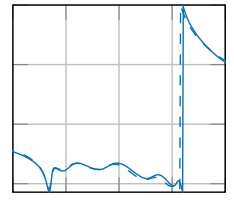

Frequence

(b) Channel 2

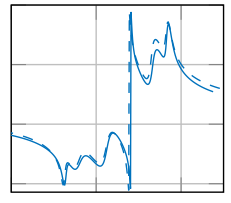

Frequence

(c) Channel 3
Fig. 5. Filter response. $S_{22}$ : blue; $S_{21}$ : red. Circuit response (solid line) vs EM simulation (dashed line).

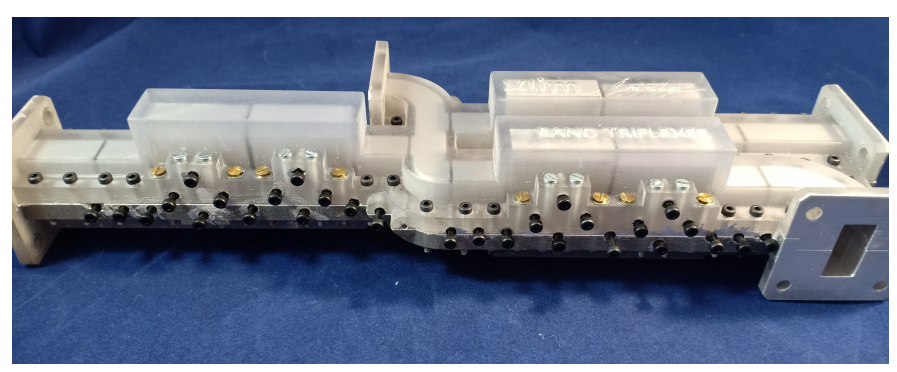

Fig. 6. Triplexer structure: plastic prototype.

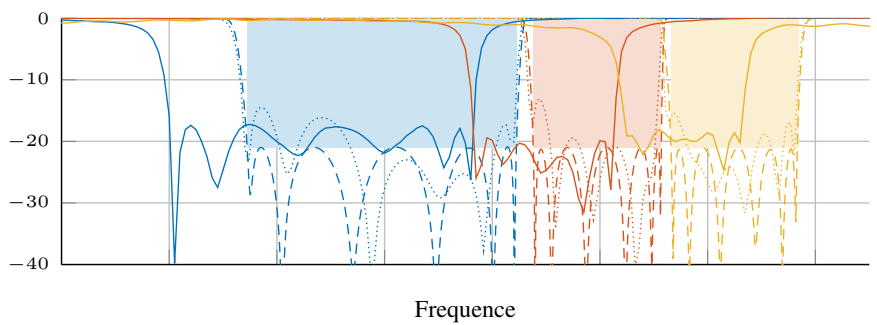

(a) Channel reflection

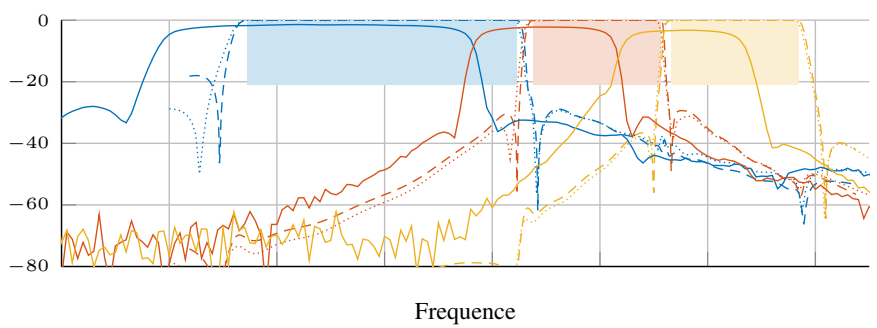

(b) Transmission toward commun port

Fig. 7. Triplexer response in dB: circuit (dashed line) vs EM simulation (dotted line) vs measurements (solid line).

\section{CONCLUSION}

A rigorous algorithm for the design of multiplexers has been presented. This algorithm allows, first to design the manifold taking into account the problem of manifold peaks, and secondly, to synthesise the channel filters through a continuation algorithm obtaining the circuit model of each filter without any overall optimization or modification of the already dimensionated manifold. Therefore, the proposed procedure leads to a decoupling of the manifold design and the synthesis of the channel filters. To illustrate the proposed technique, a waveguide triplexer is synthesized with three broadband channels. This triplexer is composed of three quasi-elliptical 6-pole filters with rectangular cavities.

\section{ACKNOWLEDGEMENTS}

This work is supported by the CNES and DGA.

\section{REFERENCES}

[1] M. Brumos, S. Cogollos, M. Martinez, P. Soto, V. E. Boria, and M. Guglielmi, "Design of waveguide manifold multiplexers with dual-mode filters using distributed models," in 2014 IEEE MTT-S International Microwave Symposium (IMS2014), June 2014, pp. 1-4.

[2] H. Hu and K. Wu, "Diagnosis and remedy of manifold spurious mode resonance in waveguide multiplexers," in Asia-Pacific Microwave Conference 2011, Dec 2011, pp. 1570-1573.

[3] L. Baratchart, M. Olivi, and F. Seyfert, "Boundary nevanlinna-pick interpolation with prescribed peak points. Application to impedance matching," SIAM Journal on Mathematical Analysis, 2017.

[4] G. Macchiarella and S. Tamiazzo, "Synthesis of star-junction multiplexers," IEEE Transactions on Microwave Theory and Techniques, vol. 58, no. 12, pp. 3732-3741, Dec 2010.

[5] E. Laplanche, A. Delage, A. Haidar, W. Feuray, J. Sence, A. Perigaud, O. Tantot, N. Delhote, S. Verdeyme, S. Bila, and L. Carpentier, "Recent development in additive manufacturing of passive hardware and conformal printing," in European Microwave Conference (EuMW 2018), Madrid, Spain, Sep. 2018. 